\title{
An unfolded protein response is the initial cellular response to the expression of mutant matrilin-3 in a mouse model of multiple epiphyseal dysplasia
}

\author{
Seema Nundlall • M. Helen Rajpar • Peter A. Bell • Christopher Clowes • \\ Leo A. H. Zeeff • Benjamin Gardner • David J. Thornton • \\ Raymond P. Boot-Handford • Michael D. Briggs
}

Received: 23 February 2010 /Revised: 19 March 2010 /Accepted: 22 March 2010 / Published online: 30 April 2010

(C) The Author(s) 2010. This article is published with open access at Springerlink.com

\begin{abstract}
Multiple epiphyseal dysplasia (MED) can result from mutations in matrilin-3, a structural protein of the cartilage extracellular matrix. We have previously shown that in a mouse model of MED the tibia growth plates were normal at birth but developed a progressive dysplasia characterised by the intracellular retention of mutant matrilin-3 and abnormal chondrocyte morphology. By 3 weeks of age, mutant mice displayed a significant decrease in chondrocyte proliferation and dysregulated apoptosis. The aim of this current study was to identify the initial post-natal stages of the disease. We confirmed that the disease phenotype is seen in rib and xiphoid cartilage and, like tibia growth plate cartilage is characterised by the intracellular retention of mutant matrilin-3. Gene expression profiling showed a significant activation of classical unfolded protein response (UPR) genes in mutant chondrocytes at 5 days of age, which was still maintained by 21 days of age. Interestingly, we also noted the upregulation of arginine-rich, mutated in early stage of tumours (ARMET) and cysteine-rich with EGF-like domain
\end{abstract}

Electronic supplementary material The online version of this article (doi:10.1007/s12192-010-0193-y) contains supplementary material, which is available to authorized users.

S. Nundlall • M. H. Rajpar · P. A. Bell $\cdot$ C. Clowes $\cdot$ B. Gardner $\cdot$

D. J. Thornton $\cdot$ R. P. Boot-Handford $\cdot$ M. D. Briggs $(\bowtie)$

Wellcome Trust Centre for Cell-Matrix Research,

Faculty of Life Sciences, University of Manchester,

Michael Smith Building, Oxford Road,

Manchester M13 9PT, UK

e-mail: mike.briggs@manchester.ac.uk

\section{A. H. Zeeff}

Bioinformatics Core Facility, Faculty of Life Sciences,

University of Manchester,

Manchester M13 9PT, UK protein 2 (CRELD2) are two genes that have only recently been implicated in the UPR. This endoplasmic reticulum (ER) stress and UPR did not lead to increased chondrocyte apoptosis in mutant cartilage by 5 days of age. In an attempt to alleviate ER stress, mutant mice were fed with a chemical chaperone, 4-sodium phenylbutyrate (SPB). SPB at the dosage used had no effect on chaperone expression at 5 days of age but modestly decreased levels of chaperone proteins at 3 weeks. However, this did not lead to increased secretion of mutant matrilin-3 and in the long term did not improve the disease phenotype. We performed similar studies with a mouse model of Schmid metaphyseal chondrodysplasia, but again this treatment did not improve the phenotype.

Keywords Matrilin-3 · Chondrodysplasia · Mouse model · Unfolded protein response . Chemical chaperones . ARMET.CRELD2

\section{Introduction}

The rough endoplasmic reticulum (rER) has stringent quality control mechanisms to ensure that only properly folded proteins are allowed to leave the rER, thus mediating the secretion of high-quality functional proteins (Kaufman 1999, 2002; Rutkowski and Kaufman 2004). Correctly folded proteins are translocated to the Golgi apparatus where they undergo additional processing before trafficking to their intended destinations, either secretion into the extracellular matrix (ECM) or embedding in the plasma membrane or to the lysosome.

A number of protein folding disorders result from the accumulation of unfolded or misfolded proteins within the 
rER, causing ER stress (Kaufman 2002). This ER stress can lead to an upregulation of many rER resident proteins involved in protein folding to protect the cells from the ER stress and is known as an unfolded protein response (UPR).

The chondrodysplasias are a clinically and genetically heterogeneous group of over 200 unique phenotypes that effect the development of the osseous skeleton (Hall 2002). Numerous chondrodysplasias can result from mutations in the genes encoding cartilage extracellular matrix proteins (Superti-Furga et al. 2001) that can sometimes lead to misfolding and an accumulation of mutant protein within the rER (Bateman et al. 2009; Boot-Handford and Briggs 2009). Mutant protein accumulation in the rER is a key pathological feature in a range of different mouse models of chondrodysplasias, primary amongst which are models of pseudoachondroplasia, multiple epiphyseal dysplasia (Leighton et al. 2007; Schmitz et al. 2008) and metaphyseal chondrodysplasia type Schmid (MCDS; Ho et al. 2007; Rajpar et al. 2009; Tsang et al. 2007).

Multiple epiphyseal dysplasia (MED) is a clinically variable and genetically heterogeneous chondrodysplasia that is characterised by mild short stature, joint pain and stiffness and early onset osteoarthritis. Autosomal-dominant forms of MED can result from mutations in the genes encoding three cartilage structural proteins, cartilage oligomeric matrix protein, type IX collagen and matrilin-3 (Briggs and Chapman 2002). Matrilin-3 is the third member of a family of four ECM proteins; matrilin-1 and matrilin-3 are primarily expressed in cartilage, whilst matrilin-2 and matrilin-4 have a wider pattern of expression in a variety of ECM including non-skeletal tissues (Wagener et al. 2005).

Matrilin-3 consists of a single von Willebrand factor A-like domain (A-domain), four epidermal growth factor (EGF)-like motifs and a coiled-coil oligomerization domain (Wagener et al. 1997). All of the MED-causing mutations in MATN3 are found in the single A-domain of matrilin-3 in either the $\beta$-strand or $\alpha$-helix regions (Fresquet et al. 2008; Jackson et al. 2004). The majority of these mutations have been shown to result in the retention of mutant protein in vitro, whereas a smaller proportion of mutations appear to allow the secretion of mutant protein (Cotterill et al. 2005; Fresquet et al. 2008).

In order to determine the precise disease mechanisms that underpin the pathophysiology of MED in vivo, we generated a murine model by introducing a diseasecausing Matn3 mutation (p.V194D). This mouse model exhibited significant short-limb dwarfism with a marked reduction in chondrocyte proliferation and dysregulated apoptosis within the cartilage growth plates of the developing limbs. Mutant matrilin-3 was retained within the rER of chondrocytes, and preliminary experiments demonstrated the upregulation of BiP and Grp94 in chondrocytes from 4-day-old mice (Leighton et al. 2007).
The aim of this current study was to use microarray, quantitative real-time reverse-transcriptase polymerase chain reaction (qRT-PCR) and Western blotting to comprehensively characterise the initial cellular response in the post-natal stage of the disease.

\section{Materials and methods}

\section{Real-time PCR and Western blot analysis}

For qRT-PCR, the rib cages from 5-day-old mice (wild type (wt) and mutant $(\mathrm{m} / \mathrm{m})$ ) were dissected and treated with collagenase (type $1 \mathrm{~A}, 2 \mathrm{mg} / \mathrm{ml}$ ) for $1 \mathrm{~h}$ at $37^{\circ} \mathrm{C}$ in Dulbecco's modified Eagle's medium (DMEM). The costal cartilage was dissected from individual ribs, and the perichondrium layer was removed. The cartilage was digested a second time with collagenase for $3 \mathrm{~h}$ to remove the collagen matrix and release the chondrocytes. The chondrocytes were passed through a cell strainer $(70 \mu \mathrm{m})$ and washed with DMEM containing 10\% FBS and following centrifugation washed again with PBS. The cell pellet was resuspended in $500 \mu \mathrm{l}$ TriZol (Invitrogen), and total RNA was isolated according to the manufacturer's instructions. First-strand cDNA was synthesised using random hexamer primers (Superscript III, Invitrogen), and qPCR was performed using the $\mathrm{SYBR}^{\circledR}$ green $\mathrm{PCR}$ protocol. Primer sequences were: BiP: $5^{\prime}$-ggcaccttc gatgtgtctcttc-3' and rev: 5'-tccatgace cgctgatcaa-3'; Grp94: $5^{\prime}$-taagctgtatgtacgecgegt-3' and rev: $5^{\prime}$-ggagatcatcggaatccacaac-3'; Calnexin: 5'-tga ttt cet ctc cet ccc ctt-3' and rev: 5'cac tgg aac ctg ttg atg gtg a-3'; Calreticulin: $5^{\prime}$-gct acg tga agc tgt ttc cga-3' and rev: $5^{\prime}$-aca tga acc ttc ttg gtg cca g-3'; Erp72: $5^{\prime}$-agt atg agc cca ggt tcc acg t-3' and rev: $5^{\prime}$-aga agt ctt acg atg gcc cac c-3'. Each experiment included 'no template' controls, was run in duplicate and had an $18 \mathrm{~S}$ RNA control. Each independent experiment was repeated three times, and the results were analysed by independentsamples $t$ test.

For Western blot analysis, chondrocytes were isolated as above, but aliquots of $2 \times 10^{5}$ chondrocytes were prepared and resuspended in $5 \times$ sodium dodecyl sulphate (SDS) loading buffer containing DTT. These protein aliquots were separated by 4-12\% SDS-polyacrylamide gel electrophoresis (PAGE; Invitrogen) then transferred to nitrocellulose membranes for Western blot analysis. Ponceau staining was used to confirm equal loading of total protein isolates.

Antibodies to key chaperones associated with the unfolded protein response were used at a dilution of either 1:500 (BiP, Grp94, Erp72 and PDI; all from Santa Cruz) or 1:100 (ATF-6 from Imgenex and Bcl-2 from Abcam). 
Histology and immunohistochemistry

Tissue samples were fixed overnight in ice-cold $10 \%$ formalin (haematoxylin and eosin (H\&E) staining and Terminal deoxynucleotidyl transferase dUTP nick-end labelling (TUNEL)) or $95 \%$ ethanol and $5 \%$ acetic acid (immunohistochemistry (IHC) and bromodeoxyuridine (BrdU)). Samples were then decalcified in 20\% EDTA and embedded in paraffin wax as described previously (Leighton et al. 2007). Antibodies used were rabbit anti-matrilin-3 (R\&D), rat anti-BrdU (Abcam) and goat anti-Grp94 (Santa Cruz). Briefly, endogenous peroxidase activity was quenched by a $\mathrm{H}_{2} \mathrm{O}_{2}$ /methanol wash, followed by hyaluronidase treatment. Samples were blocked with goat serum (for anti-matrilin-3 and anti-BrdU) or rabbit serum (for anti-Grp94) and bovine serum albumin (BSA) in phosphate-buffered saline (PBS) for $1 \mathrm{~h}$ and incubated for $1 \mathrm{~h}$ with the primary antibody (in PBS/BSA). They were then incubated for $1 \mathrm{~h}$ with the relevant secondary antibodies such as biotinylated goat anti-rabbit IgG (Dako Cytomation), biotinylated goat anti-rat (Abcam) or rabbit anti-goat (Santa Cruz) in PBS/BSA with goat/rabbit serum. Slides were then incubated with the $\mathrm{ABC}$ complex/HRP reagent for $30 \mathrm{~min}$ and developed using DAB. Samples were counterstained with methyl green and mounted with VectaMount. BrdU IHC was performed as above, except the hyaluronidase step was replaced with an antigen retrieval step (i.e. incubated in $4 \mathrm{M} \mathrm{HCl}$ for $15 \mathrm{~min}$ and then neutralised with $0.1 \mathrm{M}$ borate buffer).

Analysis of apoptosis was performed using the DeadEnd ${ }^{\mathrm{TM}}$ fluorometric TUNEL system (Promega) and visualised with a Zeiss Axiovision microscope. Nuclei were stained with 4',6-diamidino-2-phenylindole (DAPI) while apoptotic cells were stained with fluorescein isothiocyanate (FITC). Nested analysis of variance (ANOVA) was used to assess statistical significance between treated and untreated samples. A value of $p<0.05$ was considered statistically significant.

\section{BrdU labelling of proliferating cells}

Twenty-one-day-old mice were injected peritoneally with $0.1 \mathrm{ml}$ of cell proliferation labelling reagent (Amersham) per $10 \mathrm{~g}$ of weight and sacrificed after $2 \mathrm{~h}$. The dissected tibias were fixed and analysed by IHC using an anti-BrdU antibody (1:100; Abcam). The number of BrdU-labelled cells was expressed as a proportion of the total population of cells in the proliferative zone. Nested ANOVA was used to assess statistical significance between treated and untreated samples. A value of $p<0.05$ was considered statistically significant.

Ultrastructural analysis of cartilage

Ribs from 7-day-old mice were dissected and fixed overnight in $4 \%$ PFA $/ 2.5 \%$ glutaraldehyde in $0.1 \mathrm{M}$ sodium cacodylate buffer followed by three washes in $0.1 \mathrm{M}$ sodium cacodylate buffer. Samples were then incubated in a secondary fix of $3 \%$ potassium ferrocyanide buffer with $2 \%$ osmium oxide for $2 \mathrm{~h}$. They were washed in distilled water and incubated overnight for $2 \mathrm{~h}$ in $0.5 \%$ uranyl acetate at $40^{\circ} \mathrm{C}$. The samples were washed in distilled water and dehydrated in increasing concentrations of acetone: $50 \%, 70 \%, 90 \%$ and $100 \%$ acetone for $30 \mathrm{~min}$. The acetone was replaced with two changes of propylene oxide, which in turn was replaced with Spurr's resin. After several changes, the resin was polymerised by incubating at $60^{\circ} \mathrm{C}$ for $48 \mathrm{~h}$. Seventy-nanometer sections were cut and stained with $0.3 \%(w / v)$ lead citrate, and images were taken on a FEI Tecnai 12 Biotwin electron microscope, recorded on 4489 film (Kodak) and scanned using an Imacon Flextight 848 scanner (Precision Camera \& Video).

Phenotypic analysis of the skeleton

Mice at 63 days of age were sacrificed, and bone length measurements were made from whole-body radiographs. At least 20 mice were measured for both treated and control groups, and nested ANOVA was used to assess statistical significance between these two groups. A value of $p<0.05$ was considered statistically significant.

Microarray hybridisation

RNA quality was checked using the RNA 6000 Nano Assay and analysed on an Agilent 2100 Bioanalyser (Agilent Technologies). RNA concentration was quantified using a Nanodrop ultra-low-volume spectrophotometer (Nanodrop Technologies). The hybridisation cocktail was hybridised to Mouse 430_2 oligonucleotide arrays (Affymetrix) according to the manufacturers' instructions. Arrays were read using an Agilent GeneArray scanner 3000 7G and processed using Affymetrix GCOS (V1.4) software. Statistical analysis was performed using PUMA an R/Bioconductor package for Propagating Uncertainty in Microarray Analysis (http:// www.bioinf.manchester.ac.uk/resources/puma/index.html). PPLR is an $\mathrm{R}$ package that detects differential gene expression by including probe-level measurement error and calculating the probability of positive log-ratio (PPLR). A PPLR value of 1 indicates significant upregulation whilst PPLR values closer to 0.5 are not significant.

Blood collection and analysis of SPB

Whole blood was extracted from mice and pooled into 15-ml fractions, and plasma was isolated by centrifugation. Proteins were precipitated as described elsewhere (Carducci et al. 2001), and the samples were subjected to reverse-phase highperformance liquid chromatography (HPLC) on a reversephase C18 synergi $4 \mathrm{U}$ hydro $80 \mathrm{~A}$ column (Phenomenex) as 
per manufacturer's instructions. Prior to analysis, 4-sodium phenylbutyrate (SPB; special diet services, SCANBUR) and control compounds (phenylacetate and phenylacetylglutamine) were checked for purity by liquid chromatography time of flight mass spectrometry. Control samples were diluted in a range of 10-20 pmol/l for the generation of standard curves.

\section{Cell culture and transfection}

Chinese hamster ovary-B2 (CHO) cells were cultured in Ham's F-12 media supplemented with $10 \%$ FBS, 2 mM L-glutamine, $100 \mathrm{U} / \mathrm{ml}$ penicillin and $100 \mu \mathrm{g} / \mathrm{ml}$ streptomycin. Human wt matrilin-3 A-domain and the Ile192Asn mutation had previously been sub-cloned into pSecTag2A in-frame to a C-terminal c-myc epitope tag and transfected into CHO cells (Cotterill et al. 2005).

\section{SPB treatment of $\mathrm{CHO}$ cell cultures}

Transfected CHO cells were seeded into $75-\mathrm{cm}^{2}$ vented flasks at a density of $\sim 1.5 \times 10^{6}$ cells per flask. $\mathrm{CHO}$ cells were cultured in SPB (Biomol International, Exeter, UK) administered directly into the culture media at final concentrations of $0 \mathrm{mM}$ (untreated), 0.1, 0.5, 1, 5 and $10 \mathrm{mM}$. Cells were treated with SPB until confluent at which time protein samples were prepared for SDS-PAGE.

\section{Sample preparation for SDS-PAGE and Western blotting}

Conditioned media was removed, and the cells were lysed for SDS-PAGE and Western blot analysis. To ensure that equal quantities of protein were analysed, untreated and SPB-treated cells were counted using a haemocytometer and equal numbers of cells were resuspended in $1 \mathrm{ml}$ of culture media as appropriate. Media and lysate protein samples were analysed by SDS-PAGE and Western blotting as described previously (Cotterill et al. 2005).

\section{Results}

In order to obtain sufficient quantities of cartilage for qRTPCR and Western blotting studies, we decided to use rib and xiphoid cartilage, which are amenable to accurate and reproducible dissection. Therefore, in the first instance, we demonstrated that rib and xiphoid cartilage had a tissue pathology that was comparable to the tibia growth plate.

The cartilage growth plate in ribs and xiphoid is disorganised in mice harbouring the Matn 3 mutation

H\&E staining was performed on tissue sections in order to characterise the differences in growth plate morphology in the mutant mice compared to wild-type controls (Fig. 1a, b). The growth plates of both the rib and xiphoid cartilage from wt mice were well-organised at 5 and 21 days of age as expected. For example, the demarcation of the individual zones of the growth plate was clearly distinguishable, and chondrocytes in the proliferative zone were arranged in an ordered columnar organisation along the vertical axis of the growth plate (Fig. 1a, b; black boxes in left panels). However, in the growth plates from mice homozygous for the mutation $(\mathrm{m} / \mathrm{m})$, the columnar organisation of the chondrocytes within the proliferative zone was disrupted from 5 days of age, and the chondrocytes, instead of forming ordered columns, were more randomly arranged and generally disorganised (Fig. 1a, b; red circles in right panels). These observations were consistent with the tibia growth plate as described previously (Leighton et al. 2007).

Mutant matrilin-3 is retained within the chondrocytes of both rib and xiphoid cartilage

IHC staining of wild-type (wt) xiphoid and rib cartilage with an anti-matrilin-3 antibody confirmed that matrilin-3 was found exclusively within the ECM of the growth plate as expected (Fig. 1c, d). In contrast, IHC analysis of cartilage from mice homozygous for the mutation demonstrated that the majority of matrilin-3 was retained within the chondrocytes and that there was only minimal staining in the ECM. This retention, which was observed in chondrocytes from all regions of the growth plate, is evident by 5 days of age and still present at 21 days of age. These findings were again consistent with the tibia growth plate as described previously (Leighton et al. 2007).

Ultrastructural analysis of cartilage showed enlarged individual cisternae of rER within rib chondrocytes from mutant mice

The ultrastructure of rib cartilage from 7-day-old mice was studied by transmission electron microscopy (TEM; Fig. 2 and data not shown). The resting chondrocytes in the growth plates of wild-type mice were evenly distributed and

Fig. 1 Histological and immunohistochemical analysis of wild-type and mutant xiphoid and rib cartilage. H\&E staining of wild-type (wt) and mutant $(\mathrm{m} / \mathrm{m})$ a rib and $\mathbf{b}$ xiphoid cartilage growth plates from 5- and 21-day-old mice. Cells in the proliferative zone form ordered columns along the vertical axis of the GP (black boxes). In the $\mathrm{m} / \mathrm{m}$ mice by 5 days of age, the chondrocytes (red circles) of the proliferative zone are rounder and more randomly orientated compared to the wt (black box). Scale bar is $100 \mu \mathrm{m}$. IHC with an anti-matrilin-3 antibody on $\mathbf{c}$ rib and $\mathbf{d}$ xiphoid growth plates from 5-, 14- and 21-day-old mice. In wildtype mice (wt), matrilin-3 staining was seen in the cartilage ECM at all ages. In mutant mice $(\mathrm{m} / \mathrm{m})$, matrilin-3 staining was predominantly within the chondrocytes with only minimal staining present in the ECM. Intracellular staining was observed from 5 days of age and was still present by 21 days of age 


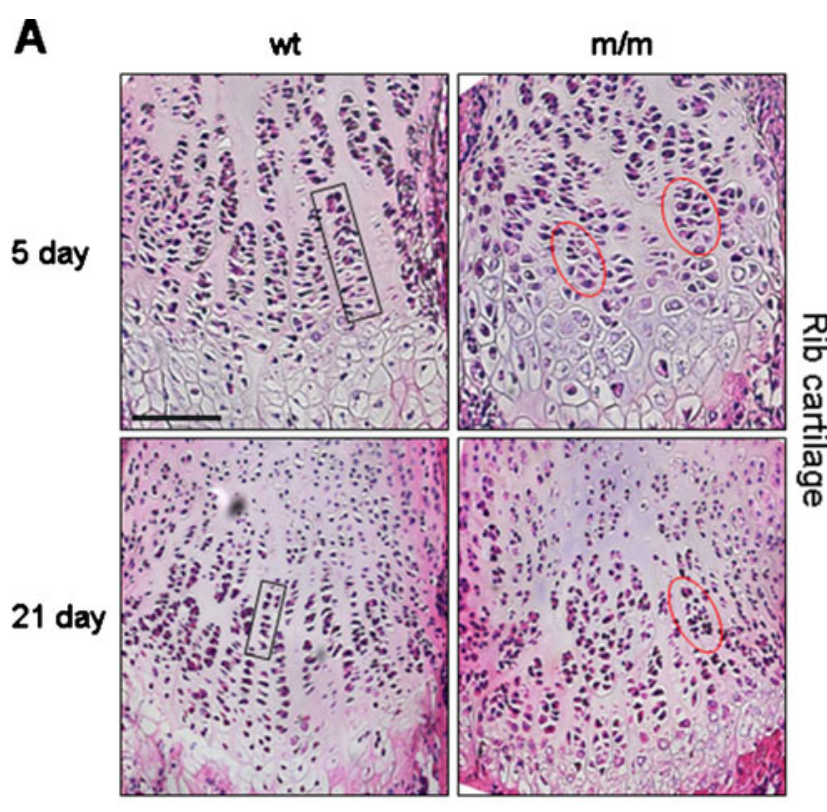

C

Rib cartilage
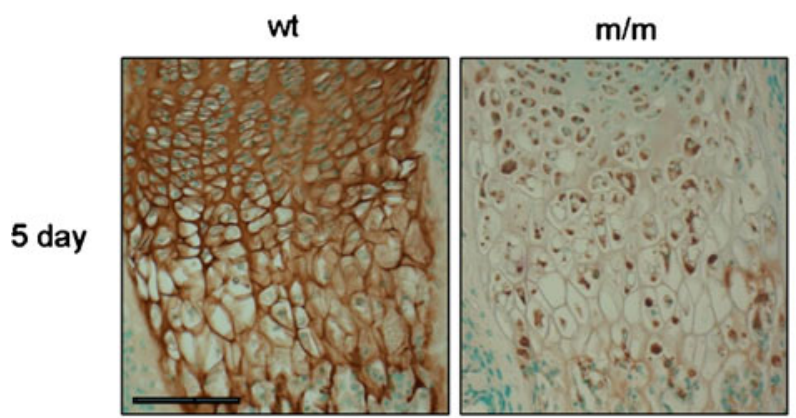

14 day
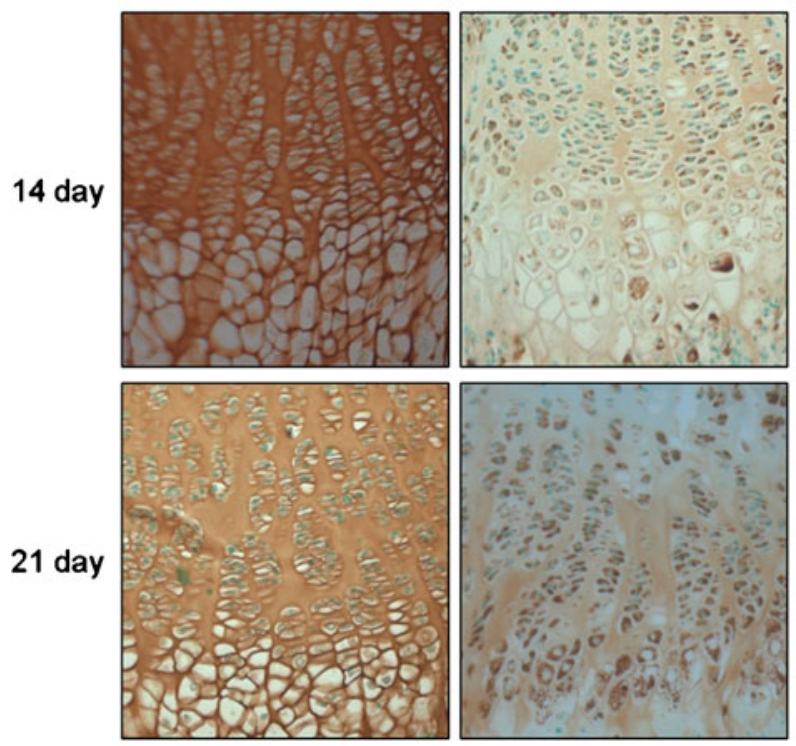

contained organised and folded cisternae of rER that appeared within normal limits (i.e. contained a limited amount of protein material that would be expected in chondrocytes actively synthesising and secreting ECM
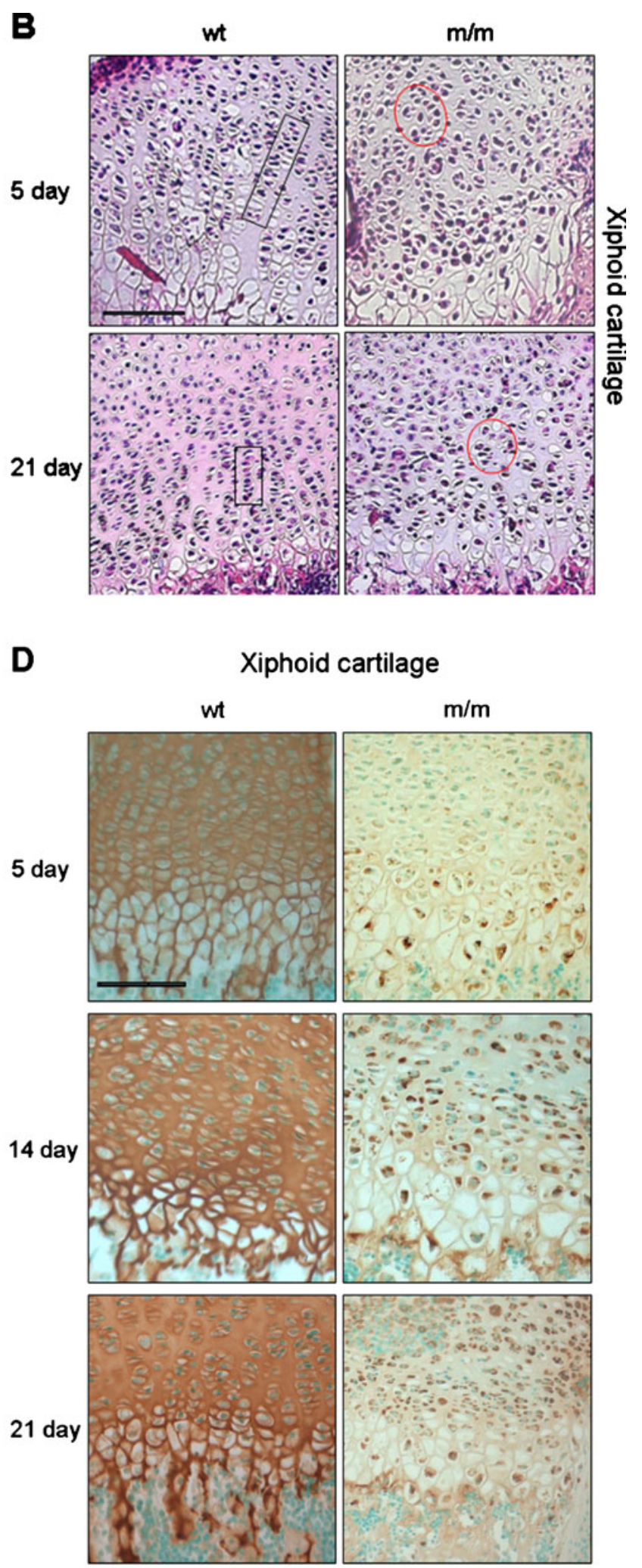

components). However, in the chondrocytes of cartilage from mutant mice, there were numerous dilated cisternae of rER that contained large amounts of amorphous material and which was confirmatory of retained protein (Fig. 2). Dilated 

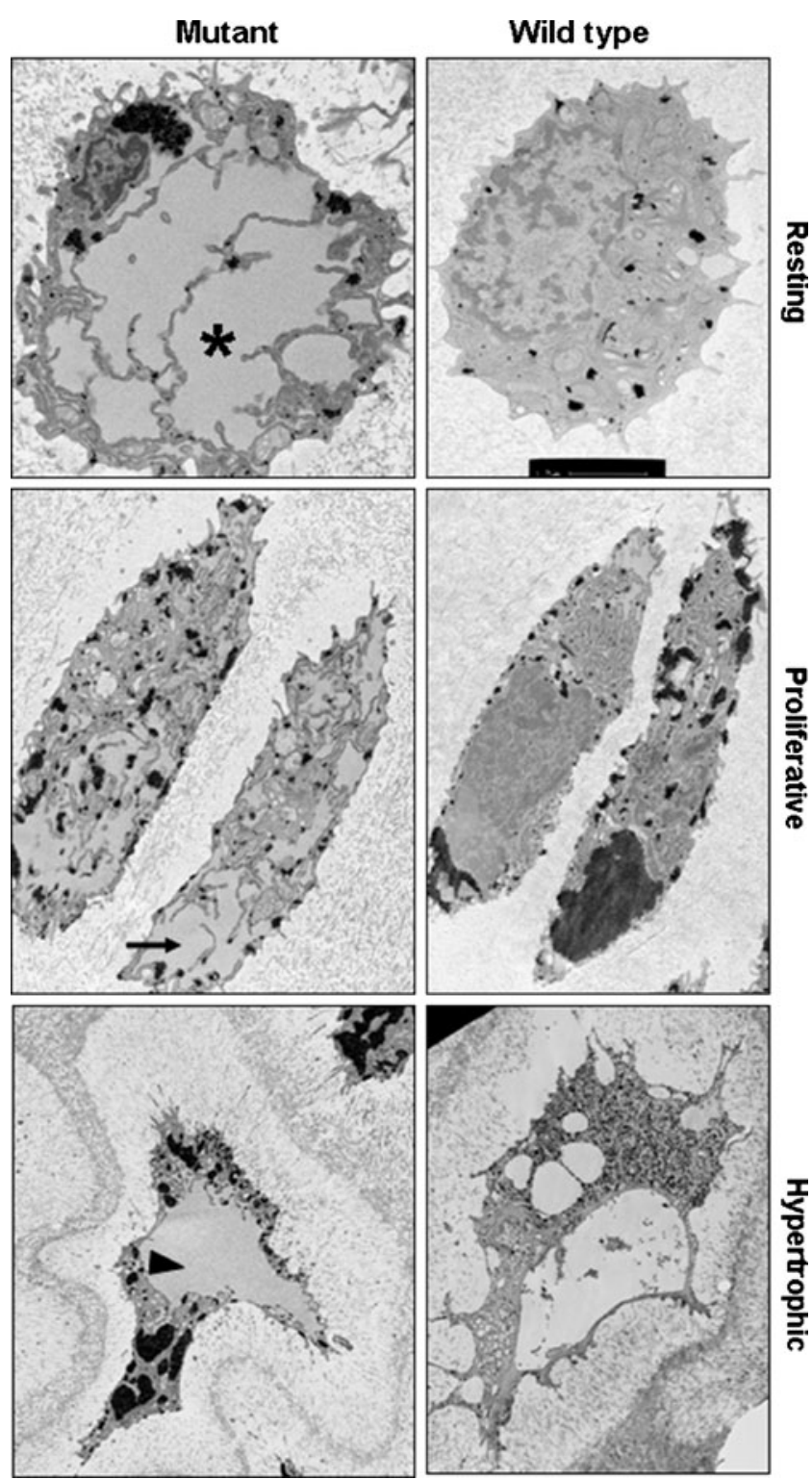

Fig. 2 Ultrastructural analysis of 7-day-old rib cartilage using TEM shows enlarged rER cisternae in mutant chondrocytes. Mutant chondrocytes in the resting (asterisk) and proliferative (arrow) zones of the growth plate have dilated cisternae of rER compared to wild-type chondrocytes from the same regions. Mutant hypertrophic chondrocytes contain the remnants of intracellular protein (arrowhead) which is not seen in wild-type hypertrophic chondrocytes

rER was seen in all of the chondrocytes of the mutant cartilage, and very few normal cisternae were present.

In the wild-type growth plate, the proliferative cells were flatter than resting chondrocytes and formed well-organised and tightly stacked two-, four- and eight-cell chondrons. However, in cartilage from mutant mice, the proliferative cells were generally slightly enlarged due to their dilated cisternae, which affected the spatial organisation of individual cells within the chondrons since they were unable to align as closely (data not shown).
The hypertrophic chondrocytes in the wild-type growth plates were generally rectangular in shape, and the condensed chromatin was clearly visible. However, in mutant mice, the hypertrophic cells were more irregular in shape, and there were clear remnants of intracellular protein (Fig. 2).

Overall, these pathological findings were consistent with those seen in the tibia growth plate as described previously (Leighton et al. 2007) and validated the use of rib and xiphoid cartilage for gene and protein expression studies. However, in characterising these tissues, we noted that after approximately 7 days of age the central part of the ribs began to ossify, which significantly reduced the number of chondrocytes that could be isolated. We therefore decided to use rib cartilage for the 5-day study and xiphoid cartilage for the 21-day study.

Gene expression profiling shows a significant activation of the UPR in mutant chondrocytes at 5 days of age that is still maintained at 21 days of age

In order to determine the differential gene expression caused by the Matn 3 V194D mutation, we chose to perform an Affymetrix genechip microarray hybridisation using RNA isolated from 5-day-old rib chondrocytes. We chose to concentrate on the 20 most significantly upregulated genes that were directly related to ER stress, UPR and/or protein folding, and these are summarised in Table 1.

In order to validate some of these microarray data, we performed qRT-PCR and Western blot analysis on a selection of genes (Fig. 3). Real-time PCR analysis confirmed a significant $\sim 2.4$-fold increase in $\mathrm{BiP}$ and a $~ 4.5$-fold increase in Grp94. In addition, ERp72, calnexin (canx) and calreticulin (calr) were also significantly upregulated by $\sim 2.7-, \sim 2.8$ and $\sim 2.8$-fold, respectively (Fig. 3a). To verify the protein levels of individual chaperones, Western blots were performed for Grp94, BiP, ERp72 and PDI, and these were found to have significant fold increases of $\sim 1.5, \sim 1.4, \sim 2.6$ and $\sim 1.3$, respectively (Fig. 3b, c). A summary of the gene and protein expression levels for the upregulated chaperones is presented in Fig. 3d. Overall, these data confirm a significant activation of the UPR in the chondrocytes of 5day-old mice homozygous for the Matn3 V194D mutation.

We also investigated the UPR in chondrocytes from the xiphoid cartilage of wild-type and mutant mice at 21 days of age. Western blots showed that there was still an increased level of ER stress in mutant chondrocytes compared to wild type. For example, BiP was upregulated by $\sim 1.3$-fold, ERp72 by $\sim 1.7$-fold and Grp94 by $\sim 3$-fold (Fig. 4a). Furthermore, IHC staining performed using an anti-Grp94 antibody showed stronger staining in the xiphoid growth plate from 21-day-old mutant mice (Fig. 4b). 
Table 1 A microarray hybridisation was performed on RNA isolated from the chondrocytes of 5-day-old rib cartilage from wild-type and mutant mice

\begin{tabular}{|c|c|c|c|}
\hline Gene and/or protein name & Gene symbol & NCBI mouse gene ID & Fold change (mut vs. wt) \\
\hline Cysteine-rich with EGF-like domains 2 & Creld 2 & 76737 & 5.77 \\
\hline Heat shock protein $90 \mathrm{kDa}$ beta (Grp94), member 1 & Grp94/Hsp90b1 & 22027 & 4.59 \\
\hline Transformation-related protein 53 binding protein 1 & $\operatorname{Trp} 53 b p 1$ & 27223 & 4.58 \\
\hline Arginine-rich, mutated in early stage tumours & Armet & 315989 & 4.29 \\
\hline Protein disulfide isomerase-associated 6 & Pdia6 & 71853 & 3.18 \\
\hline Protein disulfide isomerase-associated 4 (Erp72) & Erp $72 / P d i a 4$ & 12304 & 3.16 \\
\hline DnaJ (Hsp40) homologue, subfamily A, member 4 & Dnaja4 & 58233 & 3.06 \\
\hline Solute carrier family 35, member B1 & Slc35b1 & 110172 & 2.61 \\
\hline Serine (or cysteine) peptidase inhibitor, clade A, member $3 \mathrm{~N}$ & Serpina $3 n$ & 20716 & 2.29 \\
\hline Mannoside acetylglucosaminyltransferase 2 & Mgat2 & 217664 & 2.11 \\
\hline DnaJ (Hsp40) homolog, subfamily C, member 3 & Dnajc3 & 100037258 & 2.57 \\
\hline Signal peptidase complex subunit 3 homolog (S. cerevisiae) & Spcs3 & 317409 & 2.50 \\
\hline Heat shock $70 \mathrm{kDa}$ protein 5 (glucose-regulated protein $\mathrm{BiP}$ ) & BiP/Hspa5 & 14828 & 2.46 \\
\hline Signal peptidase complex subunit 2 homolog (S. cerevisiae) & $\operatorname{Spcs} 2$ & 66624 & 2.16 \\
\hline DnaJ (Hsp40) homologue, subfamily B, member 1 & Dnajb1 & 81489 & 2.10 \\
\hline Calnexin & Canx & 12330 & 2.10 \\
\hline Protein disulfide isomerase-associated 3 (Erp 57) & Erp57/Pdia3 & 14827 & 2.03 \\
\hline Calreticulin & Calr & 12317 & 1.95 \\
\hline $\begin{array}{l}\text { Homocysteine-inducible, endoplasmic reticulum stress-inducible, } \\
\text { ubiquitin-like domain member } 1\end{array}$ & Herpud1 & 64209 & 1.83 \\
\hline Nucleobindin 1 & Nucbl & 18220 & 1.82 \\
\hline
\end{tabular}

The relative expression levels of the 20 most upregulated genes with a relevant significance (PPLR) value of 1 are shown. PPLR is an R package that detects differential gene expression by including probe-level measurement error and calculating the probability of positive log-ratio (PPLR). A PPLR value of 1 indicates significant upregulation whilst PPLR values closer to 0.5 are not significant. The gene/protein name is the one recorded by the Affymetrix microarray software, and the NCBI geneID has been included as a reference. Note that PDI itself is not represented on the microarray chip but that the other family members Pdia2-6 are present

In order to characterise further the UPR in mutant chondrocytes at 5 days of age, we also examined the downstream mediators of the ER stress response, which included the cleavage of the ER membrane-anchored transcription factor ATF6 and the activation by splicing of transcription factor X-bp1. Both of these factors have been shown to be activated in a mouse model of PSACH-MED caused by a Comp mutation (Pirog-Garcia et al. 2007) and the former in a mouse model of MCDS caused by a mutation in col10al (Rajpar et al. 2009). We saw no evidence of the cleavage of ATF6 in either the wild-type or mutant chondrocyte protein extractions, compared to a positive control from the MCDS mouse (Supplemental Fig. S1). Furthermore, there were no differences between the splicing of X-bp1 mRNA in either wild-type or mutant chondrocytes.

ER stress does not lead to increased chondrocyte apoptosis in rib cartilage at 5 days of age

We have previously reported that there is dysregulated chondrocyte apoptosis at 3 weeks of age in the tibia growth plates of mice homozygous for the mutation (Leighton et al. 2007). We therefore wished to determine the pathological effect of ER stress and the UPR on chondrocyte viability at 5 days of age. TUNEL assays were performed to quantify the relative levels of chondrocyte apoptosis in the growth plates of ribs from wildtype and mutant mice; however, there were no significant differences in the relative numbers, or position, of TUNEL-positive cells between the different genotypes. Approximately $1.96 \%$ of cells were TUNEL positive in the hypertrophic zone of growth plates from wild type mice, whilst $1.98 \%$ of cells were TUNEL positive in the hypertrophic zone of mutant mice, but this small increase in apoptosis was not statistically significant (Fig. 5a). Furthermore, the expression of CHOP, an ER-stressmediated pro-apoptotic factor, was slightly but significantly downregulated in mutant cells at 5 (rib) and 21 (xiphoid) days of age as determined by qRT-PCR (Fig. 5b). Finally, there was no change in the antiapoptotic factor $B c l 2$ in mutant chondrocytes from ribs at 5 days of age as measured by densitometry of Western blots (Fig. 5c). 

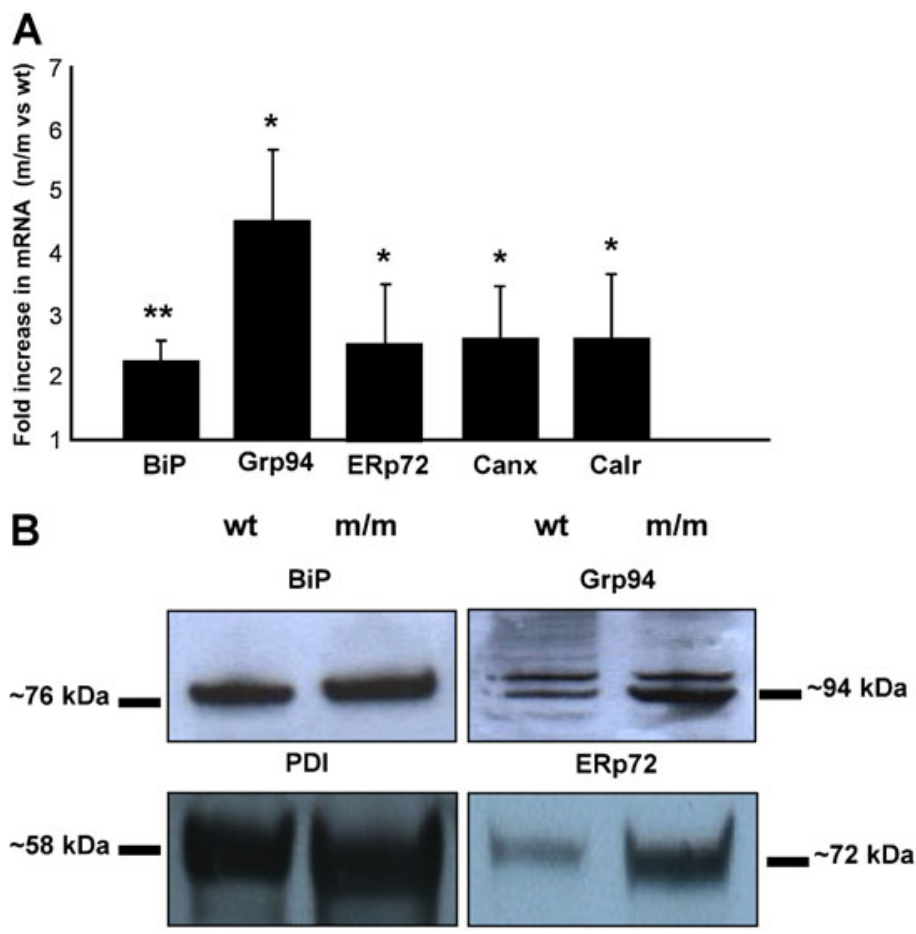

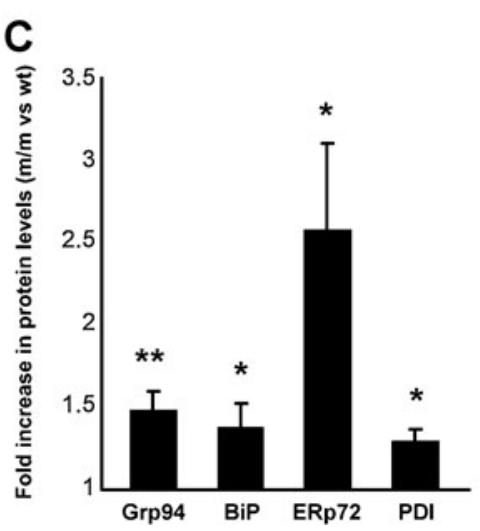

Fig. 3 qRT-PCR and Western blot validation of ER stress and UPR markers in chondrocytes from 5-day-old mutant mice. a qRT-PCR was used to validate the relative levels of gene expression in mutant $(\mathrm{m} / \mathrm{m})$ chondrocytes compared to wild-type (wt) chondrocytes. In all cases, the levels of mRNA was normalised against $18 \mathrm{~S}$ RNA. The relative levels of BiP, Grp94, ERp72, calnexin (Canx) and calreticulin (Calr) mRNA were increased between $\sim 2$-fold and $\sim 4$-fold in mutant chondrocytes $[n=3$ litters per genotype (approximately five to ten mice per litter) and three separate experiments were performed in duplicate; independent $t$ test $* p<0.05$ or $* * p<0.01)]$. b Total cellular protein was isolated from aliquots of $2 \times 10^{5}$ wt and $\mathrm{m} / \mathrm{m}$ chondrocytes and analysed by SDS-

The effect of treating mutant mice with sodium phenylbutyrate as an aid to alleviate the phenotype

Mutant mice were fed standard mouse chow pellets supplemented with SPB at a concentration of $7 \mathrm{~g} / \mathrm{kg}$ diet (effective daily consumption $\sim 1 \mathrm{~g} \mathrm{SPB} / \mathrm{kg}$ body weight) in an attempt to aid the folding and secretion of mutant matrilin-3 and alleviate the ER stress/UPR. Untreated mice
D

\begin{tabular}{|l|l|l|l|}
\hline Chaperone & $\begin{array}{l}\text { Microarray } \\
\text { (fold } \\
\text { increase) }\end{array}$ & $\begin{array}{l}\text { qRT-PCR } \\
\text { (fold } \\
\text { increase) }\end{array}$ & $\begin{array}{l}\text { Protein } \\
\text { level (fold } \\
\text { increase) }\end{array}$ \\
\hline BiP & 2.46 & 2.4 & 1.4 \\
\hline Grp94 & 4.59 & 4.5 & 1.5 \\
\hline Calr & 1.95 & 2.8 & - \\
\hline Canx & 2.10 & 2.8 & - \\
\hline ERp72 & 3.16 & 2.7 & 2.6 \\
\hline PD1 & - & - & 1.3 \\
\hline
\end{tabular}

PAGE and Western blotting using antibodies against Grp94 ( 94 kDa), BiP ( $76 \mathrm{kDa})$, ERp72 ( 72 kDa) and PDI ( $\sim 58 \mathrm{kDa})$. Western blotting consistently showed that the levels of these four proteins were increased in $\mathrm{m} / \mathrm{m}$ samples compared to wt. [ $n=3$ litters per genotype $(\sim 5-10$ mice per litter) and three separate experiments were performed in duplicate; independent $t$ test ${ }^{*} p<0.05$ or $\left.{ }^{* *} p<0.01\right)$ ]. c The Western blots were scanned and analysed by densitometry, which showed that there was a 1.5-fold increase in Grp94, 1.4-fold increase in BiP, 2.6-fold increase in ERp72 and a $\sim 1.3$-fold increase in PDI. d Comparison of the relative expression levels determined by microarray, qRT-PCR and Western blotting all showed an upregulation of key molecular chaperones

were fed with food without SPB. Quantification of blood plasma showed the presence of SPB at a concentration of $100 \mu \mathrm{mol} / 1$. Furthermore, SPB was detected in the stomachs of mice whose diet consisted of milk from their mothers who themselves were on the SPB diet (data not shown).

Microarray hybridisation was performed using RNA isolated from rib chondrocytes of 5-day-old mutant mice to 
Fig. 4 The UPR is maintained

at 21 days of age in mutant chondrocytes from xiphoid cartilage. a Western blot densitometry shows an upregulation of chaperones in chondrocytes from the xiphoid cartilage of 21-day-old mutant mice. Erp72 was upregulated by $\sim 1.8$-fold, Grp94 by 3-fold and BiP by $\sim 1.3$-fold, respectively. $[n=3$ mice per genotype and three separate experiments were performed in triplicate, independent $t$ test $* p<0.05]$. b IHC staining performed using an antiGrp94 antibody showed stronger staining in the xiphoid growth plate from 21-day-old mutant mice, particularly in chondrocytes from the proliferative zone
A

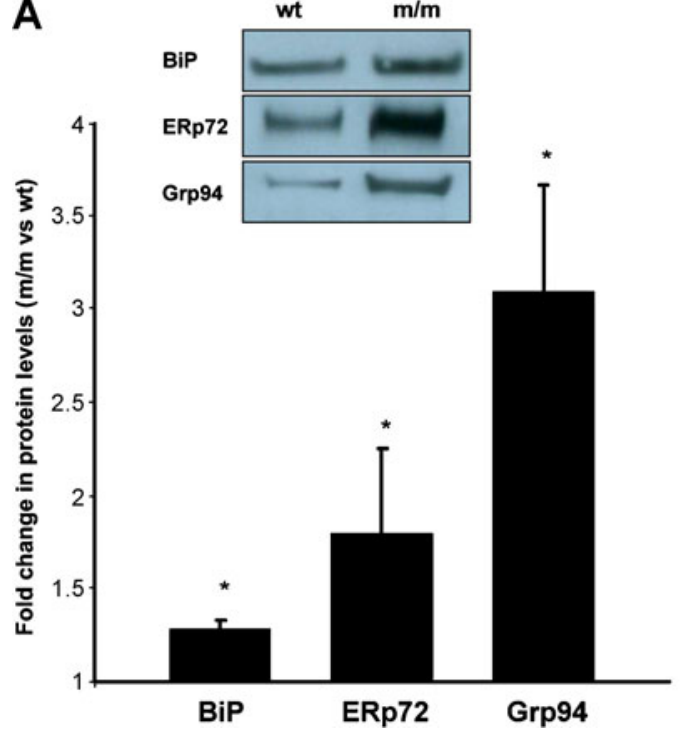

B

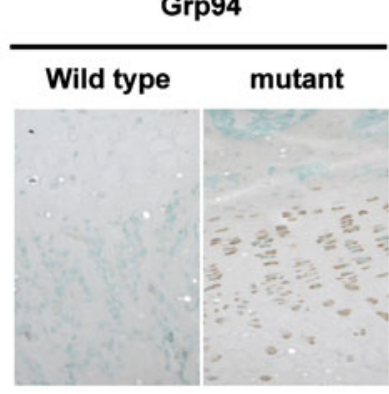

characterise the changes in gene expression following treatment with SPB (i.e. treated mutant mice vs. untreated mutant mice). The expression levels of the major chaperones BiP, Grp94, calnexin, ERp72, calreticulin and ERp57 were unchanged in treated mice compared to untreated mice (Table 2). However, at 21 days of age, the protein levels of the chaperones BiP, Grp94 and Erp72 were slightly, but significantly, downregulated by $\sim 0.74$-fold, $\sim 0.69$-fold and $\sim 0.79$-fold, respectively, in the xiphoid chondrocytes of the treated mice (Fig. 6).

Nevertheless, SPB treatment did not improve the phenotype of the mutant mice as determined by body weights and bone length measurements of 21-day-old mice, which were indistinguishable between untreated and treated mice (Fig. 7a, b). Interestingly, TUNEL assays performed on 3 -week-old tibia growth plates showed that $0.51 \%$ of cells in the hypertrophic zone were TUNEL positive in the sections from SPB-treated mutant mice compared to $0.62 \%$ in the sections from untreated mice, which equates to a $17 \%$ decrease in apoptosis (Fig. 7c). The relative levels of TUNEL-positive chondrocytes at the vascular invasion front (VIF) were $1.4 \%$ in the sections from SPB-treated mice compared to $1.1 \%$ in untreated mice (Fig. 7d). Furthermore, there was an increase in chondrocyte proliferation in the proliferative zone of growth plates from SPB-treated mice as determined by BrdU labelling. Within the proliferation zone of SPB-treated mice, $12.3 \%$ of cell nuclei were labelled with BrdU, whilst in the same region of the untreated samples only $11.4 \%$ of cell nuclei were labelled, thus representing an $8 \%$ increase in chondrocyte proliferation (Fig. 7e). However, the differences in both apoptosis and cell proliferation were not found to be statistically significant using nested ANOVA analysis. We performed similar studies with a mouse model of Schmid metaphyseal chondrodysplasia (Rajpar et al. 2009), but again this treatment did not improve the phenotype (Supplemental Fig. S2).

Finally, to determine whether treatment with SPB aids the secretion of mutant protein into the ECM, we used IHC staining on fixed sections from 3-week-old tibia growth plates. However, there was no apparent improvement in the secretion of mutant matrilin-3 in SPB-treated mice since matrilin-3 staining was indistinguishable in tibia growth plates from treated and untreated mutant mice (Fig. 7e).

We wished to determine if SPB could aid in the secretion of mutant protein from a cell culture model of mutant matrilin-3 A-domains that we have previously characterised (Cotterill et al. 2005; Fresquet et al. 2008). Increasing concentrations of SPB $(0.1$ to $10 \mathrm{mM})$ failed to aid the secretion of the mutant matrilin-3 A-domain (Supplemental Fig. S3); however, we did notice that the addition of SPB up to a final concentration of $5 \mathrm{mM}$ increased the expression of both wild-type and mutant proteins. The addition of $10 \mathrm{mM}$ SPB reduced the yields of recombinant protein, although they were still higher than without the addition of SPB (Supplemental Fig. S3) and also had a detrimental effect on cell viability regardless of whether the cells were expressing mutant or wild type matrilin-3 A-domains (not shown).

\section{Discussion}

The aim of this study was to characterise the initial cellular response to the expression of mutant matrilin-3 with the intention of identifying the key genetic pathway(s) that are ultimately responsible for decreased chondrocyte proliferation and dysregulated apoptosis at 3 weeks of age (Leighton 


\section{A}

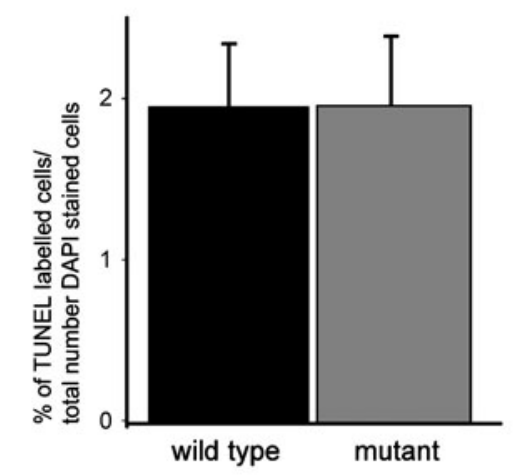

C
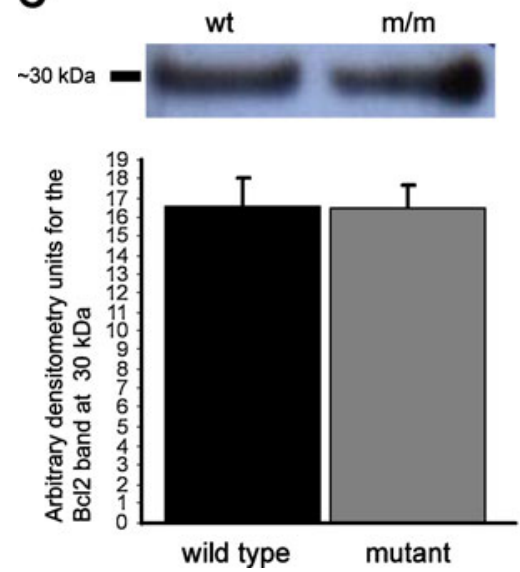

Fig. 5 TUNEL assay, CHOP and Bcl-2 analysis in chondrocytes of cartilage from wild-type and mutant mice at 5 (rib) and 21 (xiphoid) days of age. a End-stage apoptosis (DNA fragmentation) was measured in the ribs of 5-day-old mice using TUNEL assay. The relative levels of apoptosis were calculated by comparing the number of apoptotic chondrocytes (FITC-labelled nuclei) with the total number of chondrocytes in the hypertrophic zone (DAPI-labelled nuclei+FITC-labelled nuclei). TUNEL assay showed that there were no differences in the relative levels of apoptosis between mutant and wild-type chondrocytes from 5-day-old mice $[n>20$ sections per genotype, independent $t$ test]. b Chondrocytes were isolated from the ribs of 5-day-old mice and the xiphoid of 21-day-old mice, and the RNA was extracted. The relative levels of CHOP mRNA in mutant

et al. 2007). Our data from this current study demonstrate that a UPR is rapidly activated in the post-natal period, which is most likely due to accumulation of misfolded matrilin-3 within the rER of growth plate chondrocytes.

We initially demonstrated that rib and xiphoid cartilage from mutant animals displayed a tissue pathology that was similar to that previously reported for the growth plates of the major long bones (i.e. femur and tibia; Leighton et al. 2007). This disease pathology is characterised by the intracellular retention of mutant matrilin-3 from birth and a generalised growth plate dysplasia characterised by abnormal chondrocyte morphology and their misalignment

\section{B}

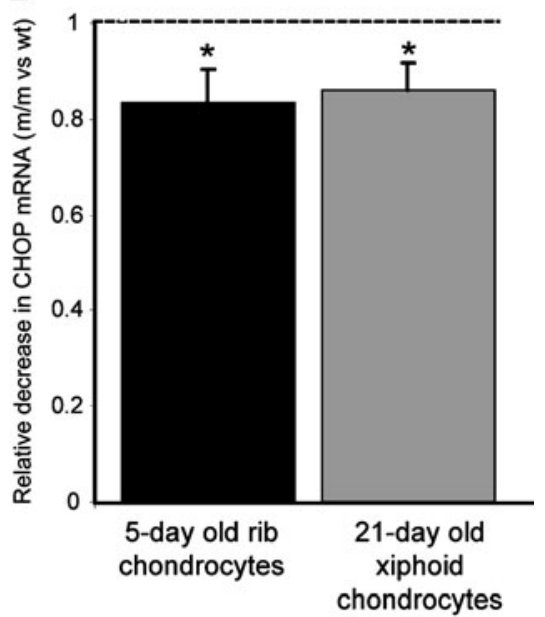

chondrocytes compared to wt chondrocytes are shown at 5 and 21 days of age. The mRNA levels were normalised against 18S RNA. There was a modest, but significant, reduction in CHOP mRNA in mutant chondrocytes. [ $n=3$ litters per genotype (five to ten mice per litter) and three separate experiments were performed in duplicate, independent $t$ test $* p<0.05]$. c Total cellular protein from 5 -day-old rib cartilage was analysed by SDS-PAGE and Western blotting with anti-Bc12. Representative Western blots show similar levels of Bcl-2 protein in mutant cells compared to wild type. Densitometry confirmed that there was no statistically significant difference in Bcl-2 levels between wild type and mutant. [ $n=3$ mice per genotype (five to ten mice per litter) and three separate experiments performed in triplicate, independent $t$ test]

within the proliferative zone. This observation suggests that weight bearing is not a factor in the expression and intracellular retention of mutant matrilin-3, which is consistent with similar findings in a pseudoachondroplastic Scottish deerhound (Stevens 1999). Our findings therefore confirm that MED is a generalised cartilage disorder (chondrodysplasia), which manifests itself most profoundly in the growth of the long bones.

The UPR that is activated in mutant chondrocytes in vivo is characterised by the upregulation of key chaperones that are known to be associated with ER stress responses or protein folding and quality control. These include BiP 
Table 2 Microarray hybridisations were performed on RNA isolated from rib chondrocytes of 5-day-old treated and untreated mutant mice

\begin{tabular}{|c|c|c|c|c|}
\hline \multirow[t]{2}{*}{ Chaperone } & \multirow{2}{*}{$\begin{array}{l}\text { Expression fold change } \\
\text { (treated vs untreated) }\end{array}$} & \multirow{2}{*}{$\begin{array}{l}\text { Significance value } \\
\text { (PPLR) }\end{array}$} & \multicolumn{2}{|c|}{ Expression levels (microarray) } \\
\hline & & & Control & Treated \\
\hline $\mathrm{BiP}$ & 1.01 & 0.52 & $6,346.856$ & $6,437.842$ \\
\hline Grp94 & 1.08 & 0.32 & 39.194238 & 36.16048094 \\
\hline Erp72 (Pdia4) & 1.04 & 0.434 & 716.4064 & 688.284 \\
\hline Calreticulin & 1.00 & 0.512 & $3,529.2909$ & $3,550.645517$ \\
\hline Erp 57 (Pdia3) & 1.06 & 0.329 & $5,520.895$ & $5,173.721$ \\
\hline Calnexin & 1.05 & 0.659 & $3,312.7712$ & $3,489.222069$ \\
\hline
\end{tabular}

There were no changes in the expression levels of key chaperone genes. $N=3$ litters (of eight to ten mice per litter) per sample (treated and untreated) mutant mice and three separate experiments were performed in duplicate. A significance (PPLR) value of 1 indicates significant upregulation whilst PPLR values closer to 0.5 are not significant

(which is a member of the Hsp70 family), Grp94, members of the Hsp40 protein family (Dnajb1, Dnajc3 and Dnaja4) and also Pdia3 (ERp57), Pdia4 (ERp72) and Pdia6. The PDI family of protein disulphide isomerase-associated proteins (PDIA2-6) are ER resident proteins that catalyse in turn the formation, reduction and isomerisation of disulphide-bonded proteins such as matrilin-3 (Hatahet and Ruddock 2007). PDI can also interact with the lectin chaperones calreticulin and calnexin, which were also increased in mutant chondrocytes. Calnexin and calreticulin bind to and mediate the folding of newly synthesised glycoproteins and are important in monitoring the N-linked glycans that persist on unfolded or misfolded glycoproteins.

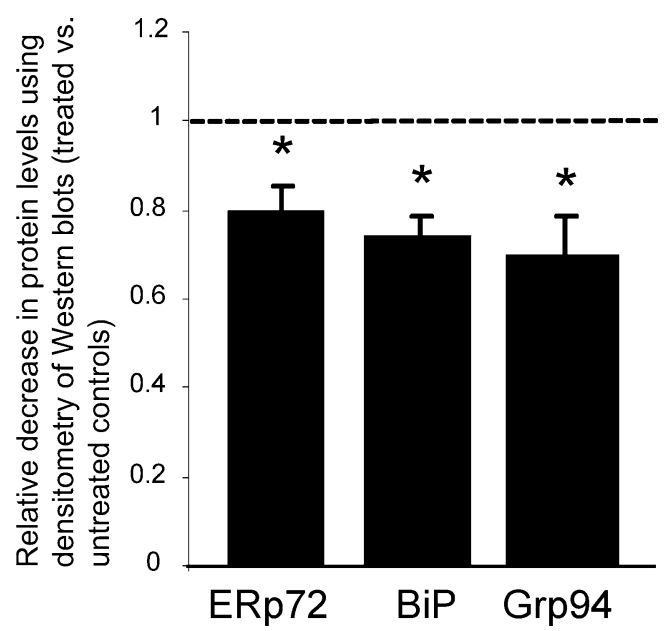

Fig. 6 Chaperone protein levels are reduced in treated mice at 3 weeks of age. Densitometry of Western blots of chondrocyte total protein from xiphoid cartilage of 21-day-old treated and untreated mutant mice. The chaperone protein levels at 21 days of age are slightly, but significantly, downregulated in the chondrocytes from the treated mutant mice. There was a $\sim 0.79$-fold decrease in Erp72, $\sim 0.74$-fold decrease in Grp78 and a 0.69-fold decrease in Grp94. $[n=3$ mice per group (treated and untreated) and two pooled cartilage samples were used in three separate experiments performed in triplicate; independent $t$ test $* p<0.05]$
The increased expression of these two genes in mutant chondrocytes might be expected since matrilin-3 has a potential N-linked glycosylation site (Wagener et al. 1997). The upregulation of Mgat 2 is further evidence that mutant matrilin-3 is being recognised and processed by the Canx/ Calr folding pathway. Mgat2 encodes a Golgi enzyme called monoacylglycerol acyltransferase 2, which catalyses an essential step in the conversion of oligomannose to complex $\mathrm{N}$-glycans, and mice deficient in Mgat2 have a congenital disorder of glycosylation type IIa (Wang et al. 2002). Furthermore, patients with the autosomal recessive disease carbohydrate-deficient glycoprotein syndrome type II have been shown to have mutations in MGAT2 (Tan et al. 1996).

We have previously shown in a cell culture model (Cotterill et al. 2005) that mutant matrilin-3 A-domains are predominantly unfolded in the rER of cells and are directly associated with ERp72 (Pdia4), a member of the protein disulphide isomerase family. This is consistent with the $\sim 3$-fold increase in the expression of Pdia 4 in mutant mouse chondrocytes (Table 1). Interestingly, in the cell culture system, no direct association was seen with PDI, BiP or Grp94, and this disparity in the chaperone protein profile between the in vitro and in vivo studies is most likely due to differences in the recognition and processing of full-length mutant matrilin-3 (this study) compared to the mutant A-domain alone (Cotterill et al. 2005). For example, the potential N-linked glycosylation site of matrilin-3 is in the second EGF-like domain between residues $\left(\mathrm{Asn}^{321}-\mathrm{Gly}^{322}-\mathrm{Ser}^{323}\right)$ and not the A-domain (Wagener et al. 1997).

In addition to chaperone/heat shock proteins and members of the lectin/PDI folding complexes, we also identified genes by the microarray studies that are known to play a role in ER-associated degradation such as homocysteine-inducible, endoplasmic-reticulum-stress-inducible, ubiquitin-like domain member 1 (Herpud1; Kim et al. 2008), suggesting that some misfolded matrilin-3 might be targeted for degradation. 
A
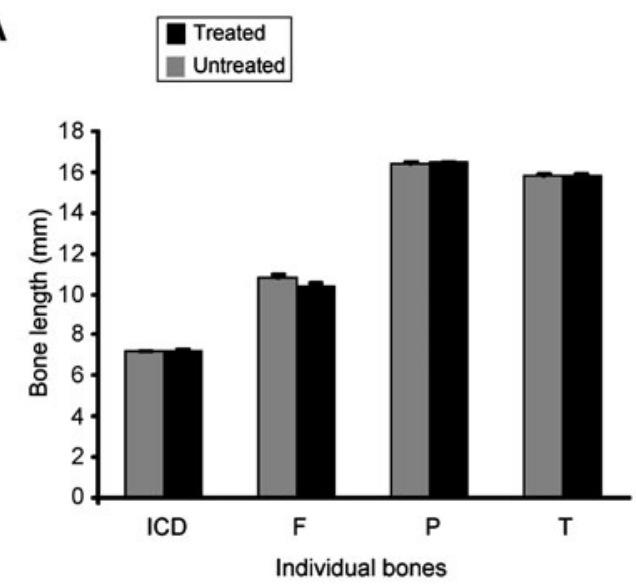

C

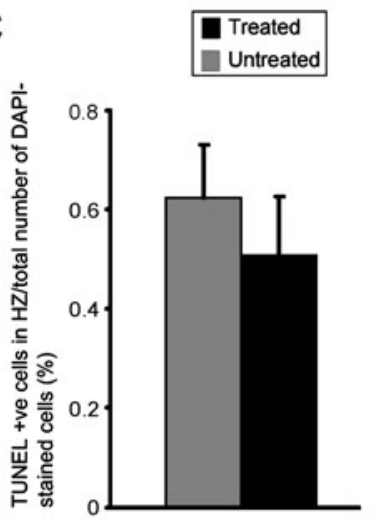

B

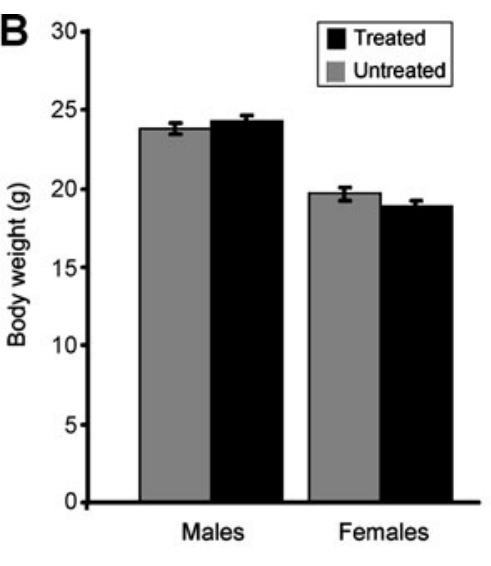

F
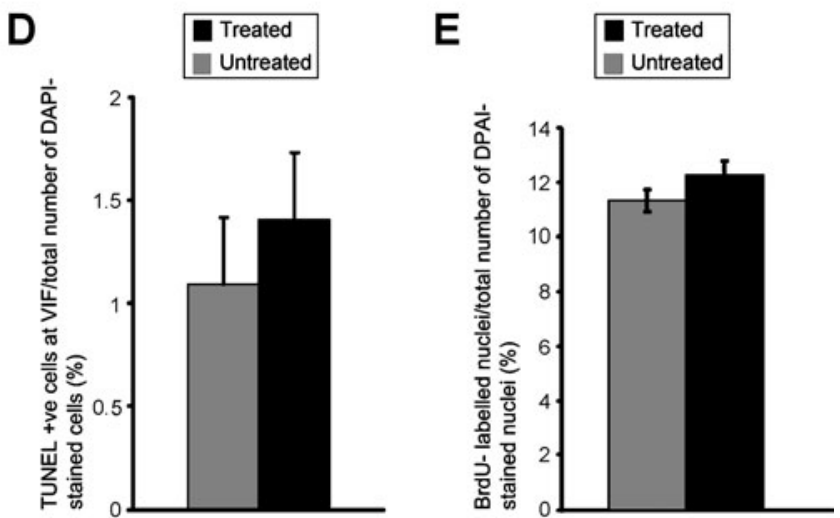

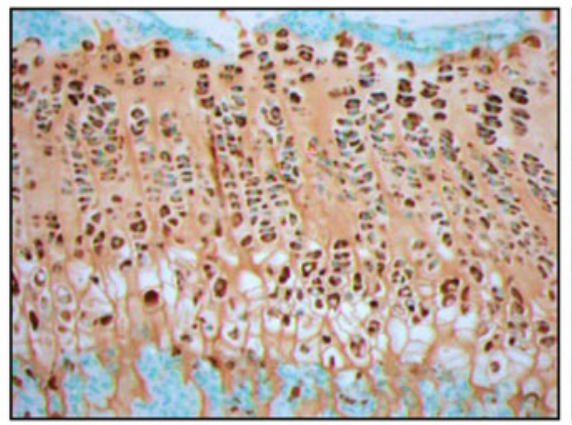

Tibia growth plate from an untreated mouse

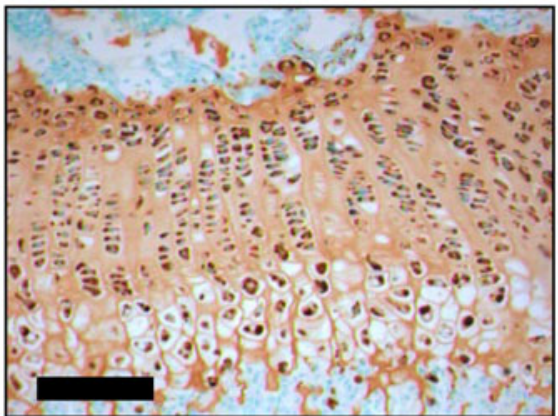

Tibia growth plate from a treated mouse
Finally, amongst the genes whose expression was upregulated in mutant chondrocytes were several genes that have only recently been implicated in ER stress. For example, cysteine-rich with EGF-like domains 2 (Creld2), which was the most highly upregulated gene in mutant chondrocytes has recently been reported as a novel ERstress-inducible gene (Oh-hashi et al. 2009). The promoter region of Creld2 contains a typical ER-stress-responsible element (ERSE) and, whilst it is believed to be involved in the folding and transport of proteins through the ER and Golgi, its precise role has yet to be determined (Ortiz et al. 2005). Another gene over-expressed to a similar extent as Creld 2 was arginine-rich, mutated in early stage of tumours (Armet; also known as MANF), which is known to be activated by the UPR via an ERSE-II element (Mizobuchi et al. 2007). Furthermore, over-expression of Armet in cell culture has been shown to inhibit cell proliferation and ERstress-induced apoptosis (Apostolou et al. 2008), both of which are key features of the growth plate dysplasia in the MED-Matn3 mouse model (Leighton et al. 2007).

We were able to determine that the post-natal activation of a UPR in mutant chondrocytes did not result in an immediate increase and/or dysregulation of CHOP-mediated apoptosis by 5 days of age. Furthermore, our data suggest that at 5 days of age the UPR is still primarily BiP-mediated and has not progressed to other downstream mediators, such as the IRE1 and PERK receptors as shown by a lack of ATF6 cleavage or $\mathrm{X}$-bp1 splicing. Unfortunately, due to technical constraints, it 
Fig. 7 The effect of SPB treatment on the phenotype of mutant mice and the relative levels of matrilin-3 retention, chondrocyte proliferation and apoptosis. a Bone length measurements of treated and untreated mutant male mice were performed on radiographs taken at 9 weeks of age showed no significant differences. Measurements were made of the inner canthal distance $(I C D)$, femur $(F)$, pelvis $(P)$ and tibia (T). ( $n>23$ mice per group; nested ANOVA). b The body weights of male and female mutant mice showed no significant differences between untreated and treated groups. ( $n>23$ mice; nested ANOVA). The relative levels of apoptosis in $\mathbf{c}$ the hypertrophic zone (HZ) and $\mathbf{d}$ at the VIF of the growth plate was calculated by counting the number of TUNEL-positive chondrocytes in the HZ or at the VIF compared to the total number of DAPI-stained chondrocytes in the growth plate. Although there appeared to be relatively less TUNELpositive cells in the $\mathrm{HZ}$ and more apoptosis at the VIF in the treated group, these differences were not statistically significant. $(n>20$ sections per group; nested ANOVA). e Twenty-one-day-old mice were administered with $0.01 \mathrm{ml} / \mathrm{g}$ of the nucleotide analogue BrdU $2 \mathrm{~h}$ prior to sacrifice. IHC was performed on tibia sections using an anti-BrdU antibody. The relative levels of chondrocyte proliferation was determined by counting the number of BrdU-labelled nuclei compared to the total number of chondrocytes in the proliferative zone of the growth plate. There were no significant differences in the proportion of proliferating cells between the treated and untreated groups. $(n>20$ sections per group; nested ANOVA). f IHC using anti-matrilin-3 antibody on tibia growth plates from 21-day-old mutant mice either treated or untreated with SPB. In both groups, there is extensive intracellular retention of mutant matrilin-3 (scale bar is $100 \mu \mathrm{m}$ )

was not possible to examine chondrocyte proliferation at this age since the mice are too small for peritoneal injection. In the long term, however, determining the precise time of onset of reduced chondrocyte proliferation and spatially dysregulated apoptosis in vivo will be important for understanding the disease process and delineating the influence of these two pathological components of MED.

We hypothesised that alleviating the rER stress and/or UPR in mutant chondrocytes may have an impact on cell phenotype and might lessen or even prevent the reduction in cell proliferation and increase spatially dysregulated apoptosis, eventually restoring normal long-bone growth. SPB is a butyrate analogue that is approved for clinical use as an ammonia scavenger in subjects with urea cycle disorders. It is also a known transcriptional regulator that may act by inhibiting histone deacetylase (Candido et al. 1978). Previous studies have shown that SPB has properties that aid the trafficking and secretion of mutant proteins in a variety of protein folding diseases such as cystic fibrosis (Rubenstein and Zeitlin 2000), hereditary hemochromatosis (de Almeida et al. 2007) and glaucoma (Tsang et al. 2007) and has been found to prevent aggregation of proteins in vitro in neurodegenerative diseases (Ryu et al. 2005). Finally, oral dosage of SPB improved the phenotype in a mouse model of type 2 diabetes (Ozcan et al. 2006) that was linked to ER cell stress (Ozcan et al. 2004).

We used a similar dosage protocol to that of Ozcan et al. (2006); however, SPB administered at this dosage in the food did not have an effect on MED disease severity as determined by body weight and bone length measurements at 9 weeks of age. Furthermore, there was no significant improvement in chondrocyte proliferation and apoptosis at 3 weeks of age. Although there appeared to be a modest increase $(\sim 8 \%)$ in chondrocyte proliferation at 3 weeks of age, this was not statistically significant, which might in part be due to the natural variability between litters. Furthermore, at 5 days of age, microarray analysis showed no changes in the expression levels of chaperones in mutant chondrocytes following treatment with SPB. Nevertheless, by 3 weeks of age, there was a modest but significant decrease in the protein levels of ERp72, BiP and Grp94, but ultimately this did not affect the trafficking of mutant matrilin-3, which remained predominately intracellular regardless of whether the mice were treated with SPB or not. SPB is known to regulate general transcription due to its role as a histone deacetylase inhibitor. The microarray results for $[\mathrm{m} / \mathrm{m}$ vs. wt] and [treated vs. untreated] had a similar overall proportion of downregulated genes with $47 \%$ of genes downregulated in $[\mathrm{m} / \mathrm{m}$ vs. wt $]$ and $43 \%$ of genes downregulated in [treated vs. untreated], suggesting that SPB did not have a major effect on the global transcription of genes in chondrocytes.

In a parallel study, we also investigated the effect of SPB treatment on a mouse model of metaphyseal chondrodysplasia type Schmid (MCDS) that we have recently described (Rajpar et al. 2009). However, like the Matn3-MED mouse, SPB treatment failed to improve the disease phenotype.

One possible reason for the SPB treatment having no effect on mutant matrilin-3 secretion, ER stress, chondrocyte proliferation and apoptosis may be due to the avascular nature of cartilage. There are no blood capillaries within cartilage, and only the perichondrium is vascularised. Instead, chondrocytes get their nutrient supply by diffusion from blood vessels of the perichondrium. Compressive loading of cartilage creates a pumping action due to the gellike and hydrated nature of cartilage that facilitates this diffusion. Since there is no readily accessible blood supply to the chondrocytes, this will limit the diffusion of SPB into the chondrocytes. Therefore, it is possible that SPB takes time to enter the chondrocytes or may not be reaching them at all. Targeting of SPB directly into cartilage may help to overcome this potential problem. However, we were unable to show that treatment with SPB could aid in the secretion of mutant matrilin-3 A-domains in vitro. Interestingly, treatment with SPB up to $5 \mathrm{mM}$ did increase the overall expression of recombinant protein and may be acting by reducing the ER stress in cells induced to over-express a recombinant protein. This finding is outside the scope of the current study but deserves further investigation.

In summary, MED is caused by a defect in the trafficking and secretion of mutant matrilin-3 that causes ER stress and a UPR in the post-natal period. Treatment with SPB may have an effect on ER stress and UPR at later stages of the disease, 
but it is too late or has insufficient efficacy to influence the disease phenotype. However, these preliminary studies using chemical chaperones will hopefully pave the way for developing suitable therapies for patients with MED and related diseases.

Acknowledgements This work was supported by grants from the Wellcome Trust (MDB is the recipient of a Wellcome Trust Senior Research Fellowship in Basic Biomedical Science; Grants 071161/Z/ 03/Z and 084353/Z/07/Z), the Arthritis Research Campaign (SN was the recipient of an ARC Ph.D. studentship) and European Commission FP6 (LSHM-CT-2007-037471). The research was undertaken in the Wellcome Trust Centre for Cell-Matrix Research and the Histology, Microarray and Transgenic Core Facilities of the Faculty of Life Sciences at the University of Manchester. Electron microscopy was performed by Roger Meadows.

Open Access This article is distributed under the terms of the Creative Commons Attribution Noncommercial License which permits any noncommercial use, distribution, and reproduction in any medium, provided the original author(s) and source are credited.

\section{References}

Apostolou A, Shen Y, Liang Y, Luo J, Fang S (2008) Armet, a UPRupregulated protein, inhibits cell proliferation and ER stress-induced cell death. Exp Cell Res 314(13):2454-2467

Bateman JF, Boot-Handford RP, Lamande SR (2009) Genetic diseases of connective tissues: cellular and extracellular effects of ECM mutations. Nat Rev Genet 10(3):173-183

Boot-Handford RP, Briggs MD (2009) The unfolded protein response and its relevance to connective tissue diseases. Cell Tissue Res 339(1):197-211

Briggs MD, Chapman KL (2002) Pseudoachondroplasia and multiple epiphyseal dysplasia: mutation review, molecular interactions, and genotype to phenotype correlations. Hum Mutat 19(5):465478

Candido EP, Reeves R, Davie JR (1978) Sodium butyrate inhibits histone deacetylation in cultured cells. Cell 14(1):105-113

Carducci MA, Gilbert J, Bowling MK, Noe D, Eisenberger MA, Sinibaldi V, Zabelina Y, Chen TL, Grochow LB, Donehower RC (2001) A Phase I clinical and pharmacological evaluation of sodium phenylbutyrate on an 120 -h infusion schedule. Clin Cancer Res 7(10):3047-3055

Cotterill SL, Jackson GC, Leighton MP, Wagener R, Makitie O, Cole WG, Briggs MD (2005) Multiple epiphyseal dysplasia mutations in MATN3 cause misfolding of the A-domain and prevent secretion of mutant matrilin-3. Hum Mutat 26(6):557-565

de Almeida SF, Picarote G, Fleming JV, Carmo-Fonseca M, Azevedo JE, de Sousa M (2007) Chemical chaperones reduce endoplasmic reticulum stress and prevent mutant HFE aggregate formation. J Biol Chem 282(38):27905-27912

Fresquet M, Jackson GC, Loughlin J, Briggs MD (2008) Novel mutations in exon 2 of MATN3 affect residues within the alphahelices of the A-domain and can result in the intracellular retention of mutant matrilin-3. Hum Mutat 29(2):330

Hall CM (2002) International nosology and classification of constitutional disorders of bone. Am J Med Genet 113(1):65-77

Hatahet F, Ruddock LW (2007) Substrate recognition by the protein disulfide isomerases. FEBS J 274(20):5223-5234

Ho MS, Tsang KY, Lo RL, Susic M, Makitie O, Chan TW, Ng VC, Sillence DO, Boot-Handford RP, Gibson G, Cheung KM, Cole
WG, Cheah KS, Chan D (2007) COL10A1 nonsense and frameshift mutations have a gain-of-function effect on the growth plate in human and mouse metaphyseal chondrodysplasia type Schmid. Hum Mol Genet 16(10):1201-1215

Jackson GC, Barker FS, Jakkula E, Czarny-Ratajczak M, Makitie O, Cole WG, Wright MJ, Smithson SF, Suri M, Rogala P, Mortier GR, Baldock C, Wallace A, Elles R, Ala-Kokko L, Briggs MD (2004) Missense mutations in the beta strands of the single A-domain of matrilin-3 result in multiple epiphyseal dysplasia. J Med Genet 41(1):52-59

Kaufman RJ (1999) Stress signaling from the lumen of the endoplasmic reticulum: coordination of gene transcriptional and translational controls. Genes Dev 13(10):1211-1233

Kaufman RJ (2002) Orchestrating the unfolded protein response in health and disease. J Clin Invest 110(10):1389-1398

Kim TY, Kim E, Yoon SK, Yoon JB (2008) Herp enhances ER-associated protein degradation by recruiting ubiquilins. Biochem Biophys Res Commun 369(2):741-746

Leighton MP, Nundlall S, Starborg T, Meadows RS, Suleman F, Knowles L, Wagener R, Thornton DJ, Kadler KE, Boot-Handford RP, Briggs MD (2007) Decreased chondrocyte proliferation and dysregulated apoptosis in the cartilage growth plate are key features of a murine model of epiphyseal dysplasia caused by a matn3 mutation. Hum Mol Genet 16(14):1728-1741

Mizobuchi N, Hoseki J, Kubota H, Toyokuni S, Nozaki J, Naitoh M, Koizumi A, Nagata K (2007) ARMET is a soluble ER protein induced by the unfolded protein response via ERSE-II element. Cell Struct Funct 32(1):41-50

Oh-hashi K, Koga H, Ikeda S, Shimada K, Hirata Y, Kiuchi K (2009) CRELD2 is a novel endoplasmic reticulum stress-inducible gene. Biochem Biophys Res Commun 387(3):504-510

Ortiz JA, Castillo M, del Toro ED, Mulet J, Gerber S, Valor LM, Sala S, Sala F, Gutierrez LM, Criado M (2005) The cysteine-rich with EGFlike domains 2 (CRELD2) protein interacts with the large cytoplasmic domain of human neuronal nicotinic acetylcholine receptor alpha4 and beta2 subunits. J Neurochem 95(6):1585-1596

Ozcan U, Cao Q, Yilmaz E, Lee AH, Iwakoshi NN, Ozdelen E, Tuncman G, Gorgun C, Glimcher LH, Hotamisligil GS (2004) Endoplasmic reticulum stress links obesity, insulin action, and type 2 diabetes. Science 306:457-461

Ozcan U, Yilmaz E, Ozcan L, Furuhashi M, Vaillancourt E, Smith RO, Gorgun CZ, Hotamisligil GS (2006) Chemical chaperones reduce ER stress and restore glucose homeostasis in a mouse model of type 2 diabetes. Science 313:1137-1140

Pirog-Garcia KA, Meadows RS, Knowles L, Heinegard D, Thornton DJ, Kadler KE, Boot-Handford RP, Briggs MD (2007) Reduced cell proliferation and increased apoptosis are significant pathological mechanisms in a murine model of mild pseudoachondroplasia resulting from a mutation in the C-terminal domain of COMP. Hum Mol Genet 16(17):2072-2088

Rajpar MH, McDermott B, Kung L, Eardley R, Knowles L, Heeran M, Thornton DJ, Wilson R, Bateman JF, Poulsom R, Arvan P, Kadler KE, Briggs MD, Boot-Handford R (2009) Targeted induction of endoplasmic reticulum stress induces cartilage pathology. PLoS Genet 5(10):e1000691

Rubenstein RC, Zeitlin PL (2000) Sodium 4-phenylbutyrate downregulates Hsc70: implications for intracellular trafficking of DeltaF508-CFTR [see comments]. Am J Physiol Cell Physiol 278:C259-C267

Rutkowski DT, Kaufman RJ (2004) A trip to the ER: coping with stress. Trends Cell Biol 14:20-28

Ryu H, Smith K, Camelo SI, Carreras I, Lee J, Iglesias AH, Dangond F, Cormier KA, Cudkowicz ME, Brown RH Jr, Ferrante RJ (2005) Sodium phenylbutyrate prolongs survival and regulates expression of anti-apoptotic genes in transgenic amyotrophic lateral sclerosis mice. J Neurochem 93:1087-1098 
Schmitz M, Niehoff A, Miosge N, Smyth N, Paulsson M, Zaucke F (2008) Transgenic mice expressing D469Delta mutated cartilage oligomeric matrix protein (COMP) show growth plate abnormalities and sternal malformations. Matrix Biol 27:67-85

Stevens JW (1999) Pseudoachondroplastic dysplasia: an Iowa review from human to mouse. Iowa Orthop J 19:53-65

Superti-Furga A, Bonafe L, Rimoin DL (2001) Molecular-pathogenetic classification of genetic disorders of the skeleton. Am J Med Genet 106:282-293

Tan J, Dunn J, Jaeken J, Schachter H (1996) Mutations in the MGAT2 gene controlling complex N-glycan synthesis cause carbohydrate-deficient glycoprotein syndrome type II, an autosomal recessive disease with defective brain development. Am J Hum Genet 59:810-817

Tsang KY, Chan D, Cheslett D, Chan WC, So CL, Melhado IG, Chan TW, Kwan KM, Hunziker EB, Yamada Y, Bateman JF, Cheung
KM, Cheah KS (2007) Surviving endoplasmic reticulum stress is coupled to altered chondrocyte differentiation and function. PLoS Biol 5:e44

Wagener R, Kobbe B, Paulsson M (1997) Primary structure of matrilin-3, a new member of a family of extracellular matrix proteins related to cartilage matrix protein (matrilin-1) and von Willebrand factor. FEBS Lett 413:129-134

Wagener R, Ehlen HW, Ko YP, Kobbe B, Mann HH, Sengle G, Paulsson M (2005) The matrilines-adaptor proteins in the extracellular matrix. FEBS Lett 579:3323-3329

Wang Y, Schachter H, Marth JD (2002) Mice with a homozygous deletion of the Mgat2 gene encoding UDP- $N$-acetylglucosamine: alpha-6-D-mannoside beta1, 2- $N$-acetylglucosaminyltransferase II: a model for congenital disorder of glycosylation type IIa. Biochim Biophys Acta 1573:301-311 